\title{
Nanoscale
}

PAPER
View Article Online

View Journal | View Issue
Check for updates

Cite this: Nanoscale, 2017, 9, 19255

\section{Poly(ferrocenylsilane) electrolytes as a gold nanoparticle foundry: "two-in-one" redox synthesis and electrosteric stabilization, and sensing applications $\dagger$}

\author{
J. Song, ${ }^{a}$ Y. N. Tan, ${ }^{* a}$ D. Jańczewski, ${ }^{b}{ }^{b}$ M. A. Hempenius, ${ }^{c}$ J. W. Xu, (D) *a H. R. $\operatorname{Tan}^{a}$ \\ and G. J. Vancso iD * $\star^{d}$
}

Gold nanoparticles (AuNPs) coated with responsive polymers gained considerable interest due to their controllable size, good stability, and fast environmental response suitable for biological applications and sensing. Here we report on a simple and efficient method for the synthesis of stable and redox responsive AuNPs using organometallic polyelectrolytes in aqueous solutions of $\mathrm{HAuCl}_{4}$. In the redox reaction, positively or negatively charged poly(ferrocenylsilanes) $\left(\mathrm{PFS}^{+} / \mathrm{PFS}^{-}\right.$) served as reducing agents, and also as stabilizing polymers. Due to their unique tunable electrostatic and electrosteric protection, AuNPs coated with $\mathrm{PFS}^{-},\left(\mathrm{PFS}^{+}\right) @$ AuNPs, possess high redox sensitivity, with reversible, repetitive, sustainable color switching between the assembled (purple color) and disassembled (red color) states as evidenced by UV-Vis absorption and TEM measurements. Feasibility studies reported here indicate that the particles described can be applied as a colorimetric probe for the detection of redox molecules, e.g. vitamin C, in a controlled and facile manner.
Received 29th June 2017, Accepted 28th October 2017 DOI: $10.1039 / c 7 n r 04697 a$ rsc.li/nanoscale and to control the interfacial properties of the AuNPs. ${ }^{10-14}$ AuNPs functionalized with a responsive polymer that is sensitive to external stimuli ( $\mathrm{pH}$, temperature, ionic strength, light, enzyme, etc.) have gained considerable interest for "smart" applications. ${ }^{15-17}$ For example, thermoresponsive poly $(N$-isopropylacrylamide) (PNIPAM) tethered AuNPs showed remarkable temperature sensitivity with a sharp, reversible clearopaque transition in solution between $25{ }^{\circ} \mathrm{C}$ and $30^{\circ} \mathrm{C} .{ }^{18}$ AuNPs protected with block copolymers (i.e. polylactic acid and polyethylene glycol, PLA- $b$-PEG- $b$-PLA) formed pH-responsive complexes. At $\mathrm{pH}=7$, these complexes were aggregated to exhibit strong near IR absorbance, while under physiological conditions, at $\mathrm{pH}=5$, the aggregates disassembled into sub $5 \mathrm{~nm}$ nanoparticles allowing for the renal clearance of the particles. ${ }^{19}$ A new class of photo-switchable AuNPs was fabricated by crosslinking of the capping material, azobenzene-modified oligonucleotides, to form aggregates, which dissociate into single discrete nanoparticles upon exposure to UV light. ${ }^{20}$ Specific protein or enzyme assisted AuNPs can be used as biological assays for nucleic acid detection, enzyme activity screening and metal ion sensing with high efficiency and selectivity. ${ }^{21-23}$ When the particles are exposed to a biological target, molecular recognition events trigger changes in molecular interactions that lead to particle responses such as assembly or aggregation. However, the reported examples often 
require complex post functionalization treatments in order to achieve a stimulus responsive effect. ${ }^{24,25}$ In addition, other concerns such as the formation of toxic byproducts during synthesis, multi-step fabrication, time consuming purification, and limited solubility in the aqueous environment ${ }^{26,27}$ frequently hamper applications. Therefore, there is a strong need for simple, facile, and well-controlled approaches to afford functional and responsive AuNPs. It is desirable to avoid, if possible, the use of additional reducing agents and surfactants, ${ }^{28}$ to perform the NP formation in aqueous media, and to directly fabricate stimulus responsive coatings during the synthesis of AuNPs using a one pot approach.

In this study, we report on a one-pot, green synthetic preparation protocol for redox stimulus responsive AuNPs using a spontaneous reduction reaction between $\mathrm{HAuCl}_{4}$ and poly (ferrocenylsilane) (PFS) polyelectrolytes. Organometallic PFS polymeric matrixes have attracted attention owing to their redox responsive behavior (originating from the ferrocene) and many unusual properties derived from the presence of Fe and $\mathrm{Si}$ atoms in the main chain. ${ }^{29,30}$ Ferrocene groups in PFS can be oxidized into the ferrocenium form using chemical agents under mild conditions. ${ }^{31-35}$ It has been reported that ferrocene containing polymers have a strong reduction capability for metal ions. For example, ferrocenyl branched poly(ethylene imine) (PEI) micelles were used as a reductive template for the preparation of AgNPs. ${ }^{36}$ Hydrogel thin films possessing ferrocenyl groups were also employed as a reducing environment for the in situ formation of metal NPs. ${ }^{37-39}$ The resulting hydrogel-PdNP composites, described in this work, were used for example in the electrocatalytic oxidation of ethanol. ${ }^{37}$ Astruc et al. reported on a new mixed-valent triazolylbiferrocenium polyelectrolyte network encapsulating AuNPs. ${ }^{40}$ In the above-mentioned examples, once the ferrocene containing polymers reduced the metal ions to synthesize the particle, the particle itself did not show further redox responsiveness. In this current article, we describe the design and use of PFS derivatives ${ }^{41}$ featuring ferrocene units in their main chain with either positively or negatively charged side groups. These PFS macromolecules serve as a reducing agent and an electrosteric stabilizer to enable the simultaneous in situ formation and stabilization of redox-responsive AuNPs in aqueous environments.

Two types of PFS polyelectrolytes with positively and negatively charged side groups in their structure were used as depicted in Fig. 1a. The reduction and stabilization of the AuNPs show differences in the electrosteric stabilization mechanism, depending on the sign of the Coulombic charge on the side groups of the polymer. It was also found that the as-synthesized PFS ${ }^{-}$(AuNPs will undergo a reversible color change from purple to red (aggregation-dispersion) in response to reducing (e.g., vitamin $\mathrm{C}$ ) and oxidizing agents (e.g., $\mathrm{FeCl}_{3}$ ) in multiple cycles. The properties, such as high colloidal stability, reversible color change, and simple assay protocol without the use of sophisticated instrumentation for sensing enable the use of PFS $^{-}$@AuNPs as a smart sensing probe for the detection of redox responsive molecules, e.g. in bio(medical) research.

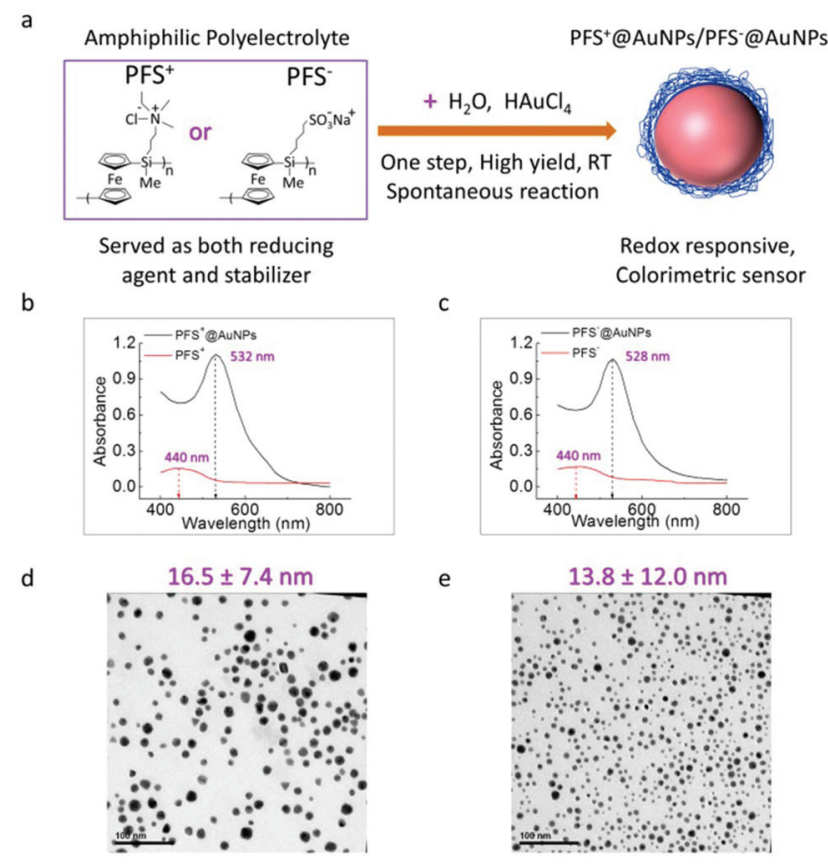

Fig. 1 (a) Molecular structure of PFS polyelectrolytes (cationic and anionic) and the schematic diagram illustrating the in situ synthetic protocol for preparing PFS@AuNPs. (b) Typical absorption spectra of $\mathrm{PFS}^{+}$(aAuNP solutions. $\mathrm{C}_{\mathrm{PFS}^{+}}: \mathrm{C}_{\mathrm{HAuCl}_{4}}=60 \mu \mathrm{M}: 1 \mathrm{mM}$. (c) Typical absorption spectra of PFS ${ }^{-}$aAuNP solutions. $C_{\mathrm{PFs}^{-}}: C_{\mathrm{HAuCl}_{4}}=60 \mu \mathrm{M}: 1 \mathrm{mM}$. (d) Typical TEM image of PFS ${ }^{+}$aAuNPs. (e) Typical TEM image of PFS $^{-}$(aAuNPs. The sizes of the NPs are shown above the TEM images. The scale bar is $100 \mathrm{~nm}$ for both TEM images.

\section{Experimental}

\section{Materials}

Hydrogen tetrachloroaurate(III) trihydrate $\left(\mathrm{HAuCl}_{4} \cdot 3 \mathrm{H}_{2} \mathrm{O}\right.$, $\geq 99.5 \%$ ), iron(III) chloride reagent $\left(\mathrm{FeCl}_{3},>97.0 \%\right)$, L-ascorbic acid (Vit C) $\left(\mathrm{C}_{6} \mathrm{H}_{8} \mathrm{O}_{6}, 99 \%\right)$ and sodium chloride $(\mathrm{NaCl})$ were purchased from Sigma-Aldrich Chemicals. Milli-Q water (18 $\mathrm{M} \Omega$ Millipore) was used as the universal solvent.

\section{PFS polyelectrolyte synthesis}

Positively and negatively charged poly(ferrocenylsilane) $\left(\mathrm{PFS}^{+}\right.$ and PFS ${ }^{-}$) with $M_{\mathrm{w}}=16.7 \times 10^{3} \mathrm{~g} \mathrm{~mol}^{-1}$ and PDI $=1.3$ were synthesized by ROP of strained, [1](3-chloropropyl)methylsilaferrocenophane, followed by side group modification as described previously. ${ }^{41}$ The symbol $\mathrm{PFS}^{+/-}$used in this study indicates either positively or negatively charged polyions, and is introduced for simplicity.

\section{AuNP preparation}

For the in situ synthesis of AuNPs, $\mathrm{PFS}^{+/-}$was added to water, followed by introducing the gold precursor $\mathrm{HAuCl}_{4}$. PFS concentrations ranged between $1 \mu \mathrm{M}$ and $60 \mu \mathrm{M}$. The polyelectrolytes were used in a vigorously stirred, mixed solution. By controlling the feedstock of gold precursor solutions and PFS polyelectrolytes, PFS capped AuNPs can be obtained by this route. 
Within a few seconds, the color of the solution changed indicating the formation of AuNPs. All measurements were conducted at room temperature $\left(25 \pm 2{ }^{\circ} \mathrm{C}\right)$.

\section{Characterization}

The synthesis of AuNPs was monitored by UV-Vis spectroscopy (TECAN Infinite M200, Switzerland). All spectra were recorded in the wavelength range of $400-800 \mathrm{~nm}$. A digital camera was used to record the color of the solution. The size and the morphology of AuNPs were investigated by transmission electron microscopy (TEM) and atomic force microscopy (AFM). TEM (JEOL JEM-2100) measurements were carried out at an accelerating voltage of $200 \mathrm{kV}$. The samples were prepared by the deposition of a few drops of sample solutions onto standard copper grids with a micropipette and dried in a vacuum at room temperature. The average particle size was determined from the TEM images with Image J software. AFM measurements were carried out with a Dimension ICON (Bruker, Germany) instrument in the tapping mode. Si tips with a cantilever spring constant of $40 \mathrm{~N} \mathrm{~m}^{-1}$ and a resonance frequency of $300 \mathrm{kHz}$ were purchased from Nanosensors (Bruker, Germany). AFM samples were prepared by casting a droplet of AuNP solution on freshly cleaved mica surfaces and dried in a vacuum at room temperature. The zeta potentials of AuNPs were measured with a ZetaPlus system (Brookhaven Instrument Corporation, USA).

\section{Salt stability test}

AuNP solutions of constant concentrations $\left(C_{\mathrm{PFS}}: C_{\mathrm{HAuCl}_{4}}=\right.$ $60 \mu \mathrm{M}: 1 \mathrm{mM}$ ) were mixed with $\mathrm{NaCl}$ at varying concentrations (ranging from $0.1 \mathrm{M}$ to $3 \mathrm{M}$ ). The AuNP salt stability was monitored by UV-Vis spectra and by TEM measurements.

\section{Colorimetric assay procedure}

$\mathrm{FeCl}_{3}$ and L-ascorbic acid (Vit C) were freshly prepared from $10 \mathrm{mM}$ stock solutions for each experiment. The solutions were used within 1 hour of preparation. A fixed percentage of gold nanoparticles (volume: $50 \mu \mathrm{L}$ ) were added to varying amounts of water in a small centrifuge tube, followed by the addition of $0.5 \mathrm{mM} \mathrm{FeCl}_{3}$ while keeping the total volume of the resulting solution constant at $90 \mu \mathrm{L}$. The solution was then pipetted into the Corning microplate within 1 minute of addition to test for its absorbance. Subsequently, varying concentrations of Vit $\mathrm{C}$ were added to the $\mathrm{FeCl}_{3}$-treated gold nanoparticle solution at a 2 min interval. Again, the total volume of the solution was fixed by adding water, to make up a constant volume of $100 \mu \mathrm{L}$. UV-Vis absorption spectra of the gold nanoparticle solutions were again recorded. Photographs were taken after each absorbance reading was obtained.

\section{Results and discussion}

AuNPs were fabricated by the direct reduction of the Au precursor in water. Fig. 1a shows variants of the water-based syn- thesis of PFS@AuNPs in a "burst" process. The reaction was performed by the rapid mixing of PFS polycation or PFS polyanion solutions (typically at concentrations of $60 \mu \mathrm{M}$ ) with a $1 \mathrm{mM}$ solution of $\mathrm{HAuCl}_{4}$ at room temperature. Within seconds, the colour of the solution changed from light yellow to dark red, indicating a fast reduction of $\mathrm{Au}^{\mathrm{III}}$ to $\mathrm{Au}^{0}$ and the formation of AuNPs. The AuNPs formed were stable in water without changing their properties for periods of months. As no additional surfactants were used, we postulate that the colloidal stability was provided by the adsorbed PFS chains at the surface of the AuNPs, as discussed later. Fig. 1b and c show the UV-Vis absorption spectra of the PFS-coated AuNP samples with a sharp, localized Surface Plasmon Resonance (LSPR) peak centered around $532 \mathrm{~nm}$ and 528 nm for PFS $^{+}$@AuNPs and PFS ${ }^{-} @ A u N P s$, respectively. In contrast, both the PFS polycation and polyanion have a weak absorption at $\lambda=440 \mathrm{~nm}$, which is the characteristic visible $d-d$ band absorbance of the ferrocene moiety. ${ }^{42}$ The TEM images (Fig. 1d and e) show that the AuNPs were well dispersed in water and indicate an average size of $16.5 \pm 7.4 \mathrm{~nm}$ for $\mathrm{PFS}^{+}$@AuNPs and $13.8 \pm 12.0 \mathrm{~nm}$ for $\mathrm{PFS}^{-}$@AuNPs.

The morphology and the size of the AuNPs were also investigated using AFM (ESI, Fig. S1†). The height values of the AuNPs on solid substrates by AFM were typically in the range of 10-14 $\mathrm{nm}$ for (PFS ${ }^{+}$@AuNPs) and 4-10 nm for (PFS ${ }^{-}$@AuNPs) which is consistent with the mean size of the NPs as observed by TEM.

AuNPs are small in size, large in specific area but have a tendency to form aggregates. ${ }^{43-45}$ To gain insight into the mechanism of AuNP formation and to study the influence of PFS concentration on the formation process, experiments with various ratios between the capping polymer and $\mathrm{HAuCl}_{4}$ were conducted. Fig. 2a shows the UV-Vis absorption spectra of AuNP solutions obtained at a fixed gold precursor concentration $\left(C_{\mathrm{HAuCl}_{4}}\right)$ at $1 \mathrm{mM}$ with varied $\mathrm{PFS}^{+}$concentrations $\left(C_{\mathrm{PFS}^{+}}\right)$from $1 \mu \mathrm{M}$ to $60 \mu \mathrm{M}$. Within a few seconds following the start of the mixing, the solution color of the $\mathrm{HAuCl}_{4}$ and $\mathrm{PSF}^{+}$reaction mixture (except for the $\mathrm{PFS}^{+}$concentration of $1 \mu \mathrm{M})$ turned purple-red, indicating the formation of stable AuNPs. No color change for $1 \mu \mathrm{M} \mathrm{PFS}^{+}$was observed due to the fact that the polyelectrolyte is insufficient to facilitate NP formation at this low concentration. With increasing $C_{\mathrm{PFS}^{+}}$from $1 \mu \mathrm{M}$ to $40 \mu \mathrm{M}$, more AuNPs are produced as evidenced by the increase in the LSPR peak intensity from 0.06 to 1.13. This is accompanied by a blue shift in the $\lambda_{\max }$ position from 543 to $532 \mathrm{~nm}$, suggesting the formation of AuNPs with a smaller size in the reaction mixture of $\mathrm{PFS}^{+}$with increasing $\mathrm{PFS}^{+}$concentration. It can also be noted that in the UV-Vis spectra the absorbance of the AuNPs obtained at a $\mathrm{PFS}^{+}$concentration of $60 \mu \mathrm{M}$ remained almost identical with the results obtained at $C_{\mathrm{PFS}^{+}}=40 \mu \mathrm{M}$, suggesting that $\mathrm{PFS}^{+}$was in excess from the $40 \mu \mathrm{M}$ concentration at the fixed gold precursor concentration of $1 \mathrm{mM}$. Similar experiments were carried out on the PFS anion in the PFS ${ }^{-}$@AuNP synthesis. No obvious color change was observed for $\mathrm{PFS}^{-}$polyanions at a concentration of $1 \mu \mathrm{M}$ (Fig. 2b). For $\mathrm{PFS}^{-}$, a gradual sharpening of the LSPR absor- 
a

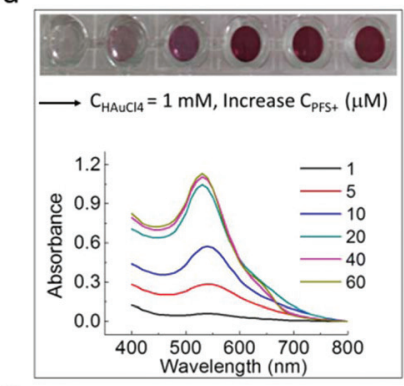

C
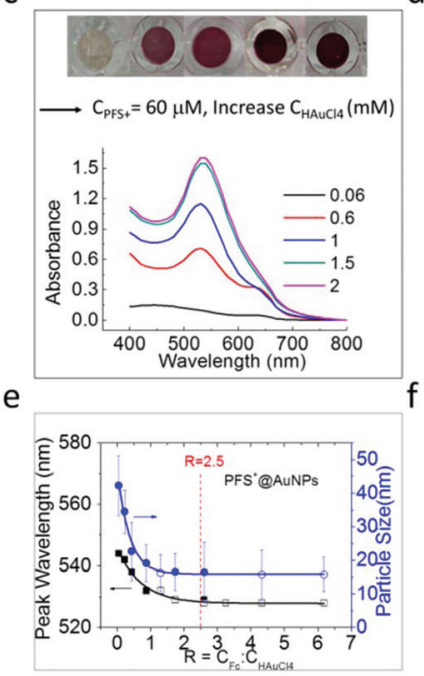

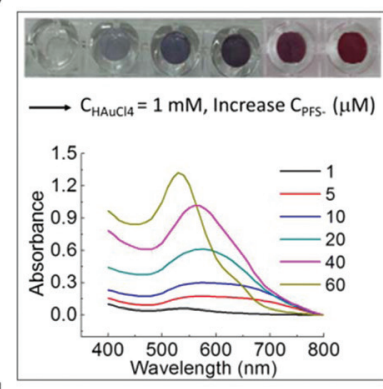

d
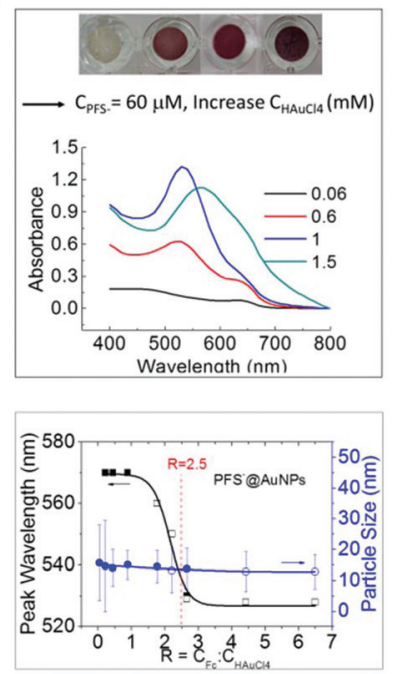

Fig. 2 (a) UV-Vis spectra of $\mathrm{PFS}^{+}$aAuNP solutions at varying $\mathrm{PFS}^{+}$concentrations, $C_{\mathrm{HAuCl}_{4}}=1 \mathrm{mM}$. Inset: Color of PFS ${ }^{+}$aAuNP solution, $C_{\mathrm{PFS}^{+}}$ from left: $1 \mu \mathrm{M}, 5 \mu \mathrm{M}, 10 \mu \mathrm{M}, 20 \mu \mathrm{M}, 40 \mu \mathrm{M}$ and $60 \mu \mathrm{M}$. (b) UV-Vis spectra of $\mathrm{PFS}^{-}$(AAuNP solutions at varying PFS ${ }^{-}$concentrations, $\mathrm{C}_{\mathrm{HAuCl}_{4}}=$ $1 \mathrm{mM}$. Inset: Color of $\mathrm{PFS}^{-}$aAuNP solution, $C_{\mathrm{PFS}}$ - from left: $1 \mu \mathrm{M}$, $5 \mu \mathrm{M}, 10 \mu \mathrm{M}, 20 \mu \mathrm{M}, 40 \mu \mathrm{M}$ and $60 \mu \mathrm{M}$. (c) UV-Vis spectra of $\mathrm{PFS}^{+} @$ auNP solutions at varying $\mathrm{HAuCl}_{4}$ concentrations, $C_{\mathrm{PFS}^{+}}=60 \mu \mathrm{M}$. Inset: Color of $\mathrm{PFS}^{+}$(aAuNP solution, $\mathrm{C}_{\mathrm{HAuCl}_{4}}$ from left: $0.06 \mathrm{mM}$, $0.6 \mathrm{mM}, 1 \mathrm{mM}, 1.5 \mathrm{mM}$ and $2 \mathrm{mM}$. (d) UV-Vis spectra of PFS- (aAuNP solutions at varying $\mathrm{HAuCl}_{4}$ concentrations, $C_{\mathrm{PFS}^{-}}=60 \mu \mathrm{M}$. Inset: Color of PFS ${ }^{-}$aAuNP solution, $\mathrm{C}_{\mathrm{HAuCl}_{4}}$ from left: $0.06 \mathrm{mM}, 0.6 \mathrm{mM}, 1 \mathrm{mM}$, and $1.5 \mathrm{mM}$. LSPR peak wavelength and diameter of (e) PFS ${ }^{+}$aAuNPs and ( $f$ ) $\mathrm{PFS}^{-}$(aAuNPs as a function of $R$ (for definition see the text). The different symbols mark the AuNPs synthesized under different reaction conditions: Solid symbols ( $\square$ and 0 ) represent the fixed precursor $\mathrm{C}_{\mathrm{HAuCl}_{4}}$ and varied $C_{\text {PFs; }}$ open symbols ( $\square$ and $O$ ) represent fixed $C_{\text {PFS }}$ and varied $\mathrm{C}_{\mathrm{HAuCl}_{4}}$. The lines are a guide to the eye (polynomial fit).

bance peaks can be seen with an increasing $\mathrm{PFS}^{-}$concentration from 5 to $40 \mu \mathrm{M}$, which is accompanied by a slight blue shift of $\lambda_{\max }$ in the range of $570-580 \mathrm{~nm}$, indicating the formation of a purplish (agglomerated as seen by the presence of precipitates) AuNP solution. A stable, red color solution of AuNPs with a sharp absorbance peak centered at $528 \mathrm{~nm}$ was observed when the $\mathrm{PFS}^{-}$concentration reached $60 \mu \mathrm{M}$.

To systematically study the effects of the variation of $C_{\mathrm{PFS}}$ to $C_{\mathrm{HAuCl}_{4}}$ ratio on the optical properties of PFS@AuNPs, we also varied the $C_{\mathrm{HAuCl}_{4}}$ while keeping the polyelectrolyte molarity constant at $C_{\mathrm{PFS}}=60 \mu \mathrm{M}$ throughout the nanoparticle synthesis for both $\mathrm{PFS}^{+}$and $\mathrm{PFS}^{-}$. In Fig. 2c, data are shown for

the $\mathrm{PFS}^{+}$polycation. It can be seen that at $C_{\mathrm{HAuCl}_{4}}=60 \mu \mathrm{M}$, there is no obvious LSPR peak of nanoparticles observed and the weak absorption signal at $\sim 440 \mathrm{~nm}$ is caused by the presence of the PFS polyelectrolyte. At $C_{\mathrm{HAuCl}_{4}}=0.6 \mathrm{mM}$, however, the absorption peak of $\mathrm{PFS}^{+} @ A u N P s$ appears at around $540 \mathrm{~nm}$. The intensity of the LSPR peak of AuNPs becomes stronger, and the absorption becomes sharper and blue shifted with increasing $C_{\mathrm{HAuCl}_{4}}$ to $1.5 \mathrm{mM}$, suggesting a higher yield and a decrease of the nanoparticle size with increasing salt concentration. At $C_{\mathrm{HAuCl}_{4}}=2 \mathrm{mM}$, the absorption peak does not change further, and remains essentially overlapped with the signal observed for $C_{\mathrm{HAuCl}_{4}}=1.5 \mathrm{mM}$ under the same reaction conditions. A weak shoulder peak in the longer wavelength region $(\sim 630 \mathrm{~nm})$ is observed due to the presence of oxidized ferrocene, i.e., ferrocenium. ${ }^{42}$ Fig. $2 \mathrm{~d}$ shows the optical absorbance for polyanionic $\mathrm{PFS}^{-}$at $60 \mu \mathrm{M}$. Here, the $\mathrm{HAuCl}_{4}$ concentration was varied from $0.06 \mu \mathrm{M}$ to $1.5 \mathrm{mM}$. When $C_{\mathrm{HAuCl}_{4}}=60 \mu \mathrm{M}$, there is an insufficient amount of the $\mathrm{HAuCl}_{4}$ precursor to allow for the formation of AuNPs, thus only a weak absorption of PFS at $\sim 440 \mathrm{~nm}$ is observed. A plasmonic signal becomes noticeable at $0.6 \mathrm{mM} \mathrm{HAuCl}_{4}$ concentration. Upon further increase of the $\mathrm{HAuCl}_{4}$ concentration up to $1 \mathrm{mM}$, the intensity of the LSPR peak of AuNPs at $\sim 530 \mathrm{~nm}$ increases. Further increase in the precursor concentration to $C_{\mathrm{HAuCl}_{4}}=1.5 \mathrm{mM}$ yielded a broad absorbance peak centered at $560 \mathrm{~nm}$. The drop in the maximum intensity indicates a certain degree of aggregation of AuNPs as confirmed by the TEM images. (ESI, Fig. S2 $\dagger$ ). Fig. 2e and f summarize the characteristics ( $\lambda_{\max }$ and $d_{\text {avg }}$ ) of PFS@AuNPs prepared under the different reaction conditions. Nanoparticle nucleation and growth is enabled by the reduction of Au ions. Since Fc is the reducing unit in both $\mathrm{PFS}^{+}$and $\mathrm{PFS}^{-}$, we considered the molar concentration ratio $C_{\mathrm{Fc}}: C_{\mathrm{HAuCl}_{4}}$ instead of $C_{\mathrm{PFS}^{+/-}}: C_{\mathrm{HAuCl}_{4}}$ as an independent variable (labeled by $R$ ) to analyze the LSPR peak wavelength and the particle size. The corresponding results are shown in the plots in Fig. 2e and f, depicting the value of $\lambda_{\max }$ and the particle size as a function of $R$. In Fig. 2e and f, solid and open symbols are used to label (a) fixed precursor and varied PFS, and (b) fixed PFS and varied precursor concentrations. These two different sets of experiments (regardless of the polyelectrolyte and precursor concentrations) show the same behavior for $\lambda_{\max }$ and for the particle size as a function of $R$. However, there are clear differences in the corresponding $\lambda_{\max }$ and NP size behavior when the pendant group charge on PFS is changed.

To better understand the relationship between the wavelength shift and the nanoparticle characteristics, TEM was used to image the morphology (ESI, Fig. S2 $\dagger$ ) and determine the average particle size $\left(d_{\mathrm{avg}}\right)$ for different $R$ values for both polycationic and polyanionic PFS. It was found that for AuNPs synthesized using $\mathrm{PFS}^{+}$, the average nanoparticle size decreased from $d_{\text {avg }}=42.3 \mathrm{~nm}$ to $d_{\text {avg }}=15.8 \mathrm{~nm}$ with an increase in $R$ values from 0.04 to 2.5 (Fig. 2e). The size of the as-synthesized PFS ${ }^{+} @ A u N P s$ remains unchanged for $R>2.5$, indicating the completion of the reaction between the polyelectrolyte and gold precursor. Thus, the blue shift of the LSPR 
peak wavelength as observed $\left(\lambda_{\max }\right)$ from $544 \mathrm{~nm}(R=0.04)$ to $532 \mathrm{~nm}(R<2.5)$ is mainly attributed to the increase in the nanoparticle size. On the other hand, for the NPs with $\mathrm{PFS}^{-}$, the particle size essentially remains constant with varying $R$, but the $\lambda_{\max }$ values shift from $570(R=0.22)$ to $530 \mathrm{~nm}$ (approximately $R=2.5$ ) and remain constant (at $528 \mathrm{~nm}$ ) for $2.5<R<6.5$. How can the constant particle size and the decrease of $\lambda_{\text {max }}$ values with increasing $R$ be understood then? To answer this question, we need to analyze the TEM images. Although the as-synthesized PFS $^{-}$@AuNPs are small in size ( $d_{\text {avg }}$ is around 12.8 to $14.6 \mathrm{~nm}$ ) when $R<2.5$, most NPs are heavily aggregated (Fig. S2(f)-(i) †). As shown in Fig. $S 2(j), \dagger$ well dispersed stabilized AuNPs were formed when $R>2.5$. From these observations, we concluded that the red shift of the LSPR peak as observed in Fig. $2 \mathrm{f}$ is mainly due to nanoparticle aggregation instead of the formation of larger AuNPs.

As both $\mathrm{PFS}^{+}$and $\mathrm{PFS}^{-}$used in this study are of similar molar mass $\left(M_{\mathrm{w}}=16.7 \times 10^{3} \mathrm{~g} \mathrm{~mol}^{-1}\right.$, PDI $\left.=1.3\right)$, i.e. a similar chain length and a similar number of ferrocene units, their reduction capability is expected to be comparable. ${ }^{46}$ However, the role of the polyelectrolyte in the nanoparticle formation and stabilization process is dependent on the sign of the side group charge ( + or - ). Based on the data we discussed above, we propose two mechanisms for the nanoparticle formation and stabilization depending on the Coulombic charge on the PFS side groups (Scheme 1).

A general chemical reduction of metallic cations to form NPs involves induction, nucleation and particle growth stages. ${ }^{28,47-49}$ A similar mechanism can be considered for the synthesis of AuNPs using PFS as the two-in-one chemical reagent (two-in-one, as PFS has a reducing and an electrosteric stabilizing function). Firstly, the ferrocene units in the polymer main chain would donate electrons to the gold precursor, $\mathrm{AuCl}_{4}{ }^{-}$, generating neutral gold atoms (by reducing $\mathrm{Au}^{3+}$ to $\mathrm{Au}^{0}$ in stepwise reactions) while Fc would become oxidized and converted into positively charged ferrocenium species. Gold NP nuclei are then formed by clustering of the gold atoms. Secondly, the polyelectrolytes are adsorbed on the surface of the gold nuclei, providing colloidal stabilization. However, prior to reaching completion of the redox reaction (oxidation of all available Fc units), the NPs continue growth while $\mathrm{Au}^{0}$ is added until there is no more available $\mathrm{Fc}$ for $\mathrm{Au}^{3+}$ reduction. Thirdly, AuNPs are stabilized by a polymer capping process of the adsorbed polyelectrolytes.

The side chains of the $\mathrm{PFS}^{+}$and the polymer are positively charged. Upon reduction of the gold precursor by the ferrocene units, the oxidized $\mathrm{PFS}^{+}$becomes more positively charged and can adsorb additional $\mathrm{AuCl}_{4}{ }^{-}$through electrostatic interactions while stabilizing the AuNPs. The surface charge of $\mathrm{PFS}^{+}$@AuNPs measured with a zeta potential analyzer is $25.3 \pm$ $4.9 \mathrm{mV}$ (for $R>2.5$ ). Since the capping layer of PFS ${ }^{+}$@AuNPs is positively charged, it has a very thin layer of steric protection as no additional $\mathrm{PFS}^{+}$in the solution can further adsorb onto this layer due to charge repulsion. As shown earlier, $\mathrm{PFS}^{+}$has a good capability to stabilize the nanoparticles formed even at low polymer concentrations. We observed that the size of the
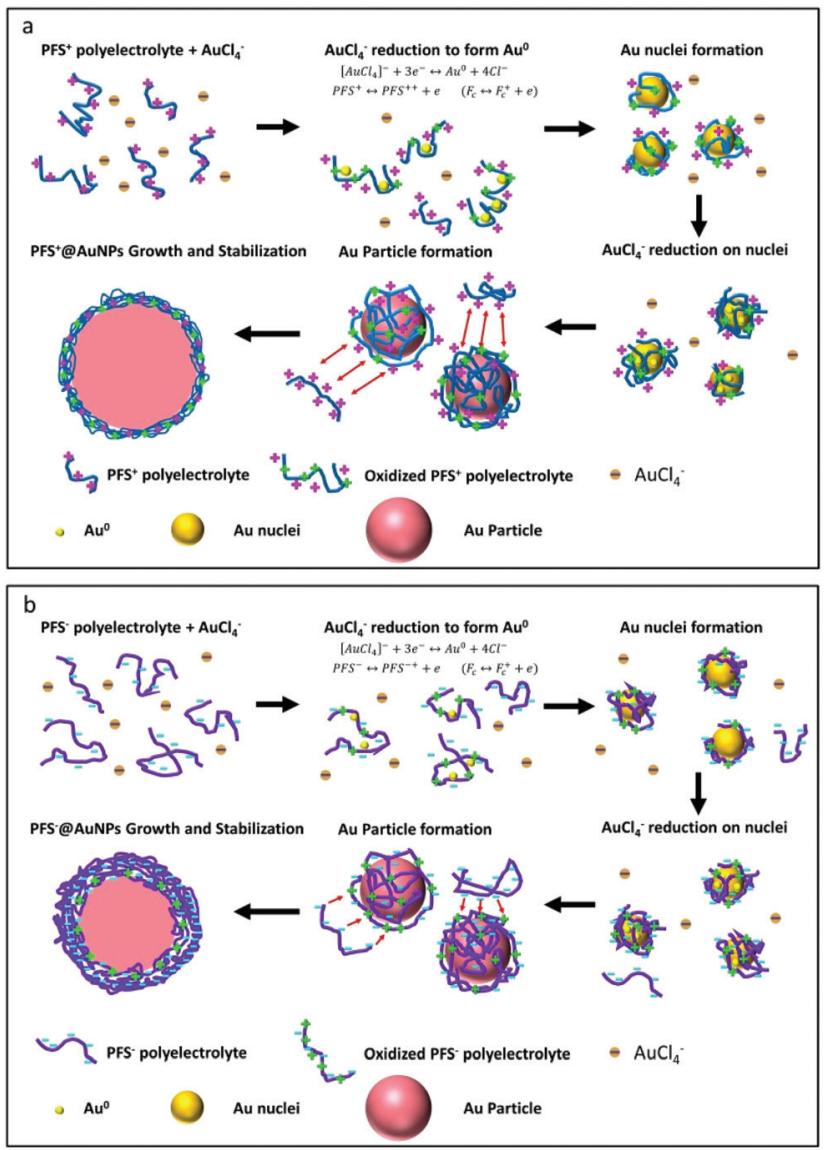

Scheme 1 (a) Proposed mechanism of AuNP formation and stabilization using polycation $\mathrm{PFS}^{+}$and (b) polyanion $\mathrm{PFS}^{-}$.

PFS $^{+} @ A u N P s$ decreases with the increase of the concentration of $\mathrm{PFS}^{+}$. This can be explained assuming that the capping capability of $\mathrm{PFS}^{+}$competes against the adsorption of $\mathrm{AuCl}_{4}{ }^{-}$, which determines the final size of the gold nanoparticles.

In contrast, the negatively charged side chains on $\mathrm{PFS}^{-}$may cause repulsion of the precursor $\mathrm{AuCl}_{4}{ }^{-}$ions during the induction stage of the particle growth, even though the precursor ions are still able to become reduced by the surrounding ferrocene of the polymer side chain to form AuNPs. The oxidized PFS $^{-}$main chains, which contain ferrocenium units, would introduce additional positive charge to the backbone and promote aggregation of $\mathrm{PFS}^{-}$@AuNPs due to charge neutralization. This electrostatic neutralization of the Au-PFS particles can be destabilized especially at low $\mathrm{PFS}^{-}$concentrations (for $R<2.5$ ). However, at the high $\mathrm{PFS}^{-}$concentration, free (unoxidized) $\mathrm{PFS}^{-}$can adsorb onto the oxidized layer of $\mathrm{PFS}^{-}$covered on the AuNP surface, giving rise to a thicker layer of polymer coating. The zeta potential of the as-synthesized PFS ${ }^{-} @ A u N P s$ was in this case $-3.76 \pm 4.7 \mathrm{mV}$, which is lower than that measured for the PFS ${ }^{+}$auNPs synthesized under the same reaction conditions $\left(C_{\mathrm{PFS}^{-}}: C_{\mathrm{HAuCl}_{4}}=60 \mu \mathrm{M}: 1 \mathrm{mM}\right)$. This result suggests that the good stability of PFS $@$ @AuNPs at a high polyelectrolyte concentration $(R>2.5)$ is mainly attributed to the electrosteric protection. 
To further confirm the nanoparticle growth and stabilization mechanisms as proposed above, we investigated the effect of ionic strength on the stability of the AuNPs in solution. Fig. 3a-c show the representative TEM images of $\mathrm{PFS}^{+}$@AuNPs with various $\mathrm{NaCl}$ concentrations. The particles were obtained at $R=2.5$ (i.e., $60 \mu \mathrm{M} \mathrm{PFS} / 1 \mathrm{mM} \mathrm{HAuCl}_{4}$ ). Aggregations can be observed on the TEM images for PFS $^{+}$@AuNPs with $0.1 \mathrm{M} \mathrm{NaCl}$ (Fig. 3b) and $3 \mathrm{M} \mathrm{NaCl}$ (Fig. 3c), respectively, which was further confirmed by UV-Vis absorption spectra (Fig. 3g). The red shift of $\lambda_{\max }$ from $530 \mathrm{~nm}(0 \mathrm{M}$ $\mathrm{NaCl})$ to $620 \mathrm{~nm}(3 \mathrm{M} \mathrm{NaCl})$ and the drop in the maximum intensity also indicate the aggregation of $\mathrm{PFS}^{+}$@AuNPs in $\mathrm{NaCl}$.

Salt induced aggregation is a well-known process in colloidal metal nanoparticle suspensions. High concentrations of salts in aqueous solution can decrease the screening length of charged FFS $^{+} @$ AuNPs thus limiting sufficient repulsion to stabilize AuNPs. Consequently, instantaneous and irreversible aggregation can happen. ${ }^{50}$ In addition, this salting-out effect increases the contribution of hydrophobic interactions between the capping PFS layers of the AuNPs and can induce aggregation. ${ }^{51}$ PFS $^{+}$@AuNPs is thus mainly protected by the electrostatic repulsion forces. On the contrary, $\mathrm{PFS}^{-}$@AuNPs synthesized under $C_{\mathrm{PFS}^{-}}: C_{\mathrm{HAuCl}_{4}}=60 \mu \mathrm{M}: 1 \mathrm{mM}$ remain stable in salt stability tests. The representative TEM images of PFS $^{-}$@AuNPs with various $\mathrm{NaCl}$ concentrations are shown in Fig. 3d-f. No obvious color change was observed from the welldispersed $\mathrm{PFS}^{-}$@AuNPs with $0.1 \mathrm{M}$ and $3 \mathrm{M} \mathrm{NaCl}$. It can also be noted in the UV-Vis spectra (Fig. 3h) that the $\lambda_{\max }$ of PFS $^{-}$@AuNPs obtained without NaCl remained almost identical with the results obtained with $0.1 \mathrm{M}$ and $3 \mathrm{M} \mathrm{NaCl}$, suggesting that $\mathrm{PFS}^{-}$@AuNPs were stable at high salt concentrations. This observation corroborates our proposed particle growth mechanism and supports the hypothesis that the thick PFS $^{-}$layers indeed play an important role in providing additional steric protection to the particles and their negative charges are not screened by the added salt.

Over the past few years, several studies based on the metal nanoparticle aggregation method has been demonstrated as

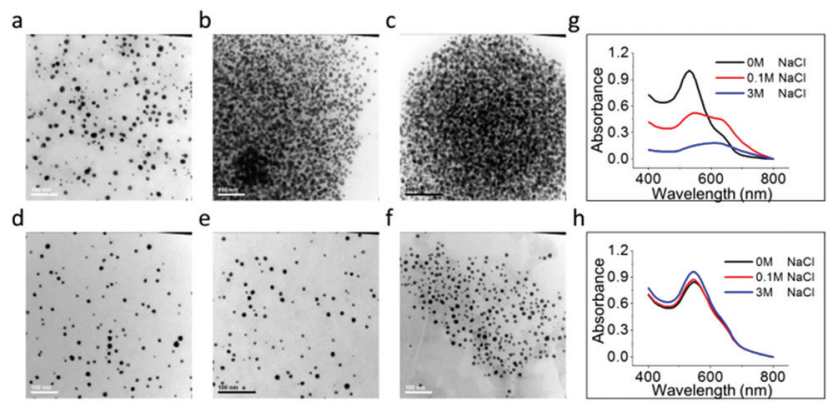

Fig. 3 TEM images of (a) $\mathrm{PFS}^{+}$(AAuNPs without $\mathrm{NaCl}$; (b) with $0.1 \mathrm{M}$ $\mathrm{NaCl}$; (c) with $3 \mathrm{M} \mathrm{NaCl}$; (d) PFS ${ }^{-}$(aAuNPs without $\mathrm{NaCl}$; (e) with $0.1 \mathrm{M}$; (f) with $3 \mathrm{M} \mathrm{NaCl}$; (g) UV-Vis spectra of PFS ${ }^{+}$aAuNP solutions at varying $\mathrm{NaCl}$ concentrations; (h) UV-Vis spectra of $\mathrm{PFS}^{-}$(aAuNP solutions at varying $\mathrm{NaCl}$ concentrations. Synthesis conditions: $C_{\mathrm{PFS}}=60 \mu \mathrm{M}$; $C_{\mathrm{HAuCl}_{4}}=1 \mathrm{mM} \mathrm{HAuCl}_{4}$. The scale bar is $100 \mathrm{~nm}$ for all TEM images. colorimetric assays for detection of various analytes such as Vc, alkaline phosphatase, etc. ${ }^{52-54}$ Combining the redoxresponsive properties of the PFS polymer and the interparticle distance dependent SPR properties of AuNPs, we propose the application of $\mathrm{PFS}^{-}$@AuNPs for the detection of reversible redox reactions with a colour change as a readout signal to indicate the concentration of the analyte. To this end, we investigated the suitability of well-dispersed $\mathrm{PFS}^{-}$@AuNPs as a colorimetric probe for the detection of the oxidizing agent $\left(\mathrm{FeCl}_{3}\right)$ and the reducing agent, ascorbic acid, (Vit $\left.\mathrm{C}\right)$ in homogeneous solutions. The assay principle is schematically illustrated in Fig. 4a. We argue that PFS @AuNPs possess a relatively thick polymer coating including both oxidized and nonoxidized $\mathrm{PFS}^{-}$. Under the assay conditions, the $\mathrm{FeCl}_{3}$ oxidizes the excess of non-oxidized ferrocene units in the polymer, thus creating more domains which are positively-charged, while the other side chains remain negatively-charged. This results in the aggregation of AuNPs. Fig. 4b shows the red-shift in the UV-Vis spectra of PSF $^{-}$@AuNPs (as the control solution) upon mixing with $0.5 \mathrm{mM}$ of $\mathrm{FeCl}_{3}$ accompanied by an immediate color change of the nanoparticle solution from red (i) to purple (ii) (see color photographs in the inset of Fig. 4b). The PFS @AuNPs in the presence of $\mathrm{FeCl}_{3}$ were found to be heavily aggregated as seen from the TEM image (ii) in Fig. 4a, as compared to the $\mathrm{PSF}^{-}$@AuNPs in control (i) which are well-dispersed in the solution. In contrast, when Vit $\mathrm{C}$ which was added subsequently into the $\mathrm{FeCl}_{3}$-treated $\mathrm{PFS}^{-}$ox@AuNP solution, a reverse colour change from purple (ii) to red (iii) (see color photographs in the inset of Fig. 4b) was observed. A blue-shift of the absorbance maximum back to the control
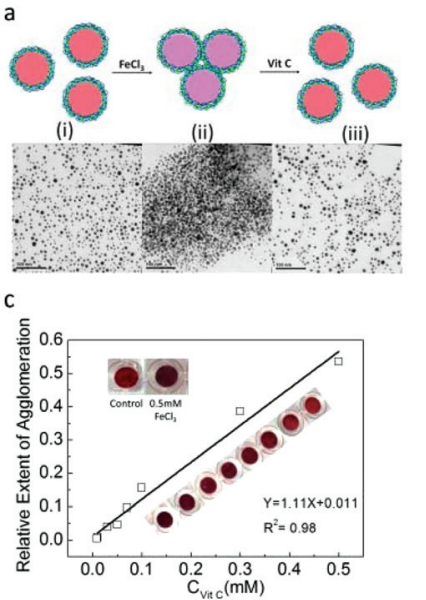

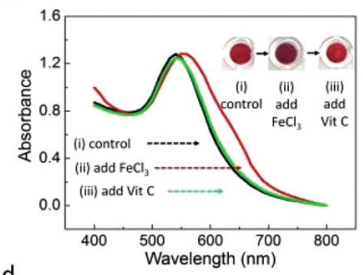

d

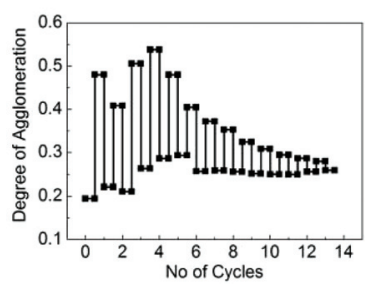

Fig. 4 (a) Schematic illustration of the assay principle for detecting oxidizing $\left(\mathrm{FeCl}_{3}\right)$ and reducing (Vit $\mathrm{C}$ ) agents using $\mathrm{PFS}^{-}$(aAuNP colorimetric probes, and TEM images of the (i) PFS ${ }^{-}$(aAuNPs (control), (ii) after the addition of $0.5 \mathrm{mM} \mathrm{FeCl}_{3}$ to the PFSox (AAuNPs, and (iii) after the addition of $0.5 \mathrm{mM}$ Vit $\mathrm{C}$ to the $\mathrm{FeCl}_{3}$ treated Vit $\mathrm{C}$ solution. (b) UV-Vis spectra of (i), (ii), (iii); inset $2 \mathrm{~b}$ shows the sequential color changes of the respective PFS ${ }^{-}$(aAuNP solution (from $\mathrm{i}$ to iii). (c) Plot of the relative extent of aggregation (induced by the fixed concentration of $\mathrm{FeCl}_{3}$ ) as a function of varying concentration of Vit C. (d) Repeatability test of the PFS $^{-}$aAuNP colorimetric assay test. 
(i) is seen as well, suggesting that the aggregated nanoparticles are now re-dispersed in the solution, as also evidenced by the TEM image displayed (iii) in Fig. 4a. The Vit $\mathrm{C}$ reduces the ferrocenium ion in the previously oxidized polymer back to its neutral form. In the reduced form of the polymer, the positively charged domains are absent, causing the particles to have weaker interactions. As a result, the particles become redispersed in the solution. Thus the color change of the colorimetric assay is regulated by aggregation/redispersion, which is a function of the redox active species concentration.

In the colorimetric assay for the detection of Vit C, $0.5 \mathrm{mM}$ of $\mathrm{FeCl}_{3}$ was first added to a series of samples containing a fixed amount of PFSox ${ }^{-}$@AuNPs, followed by the progressive addition of Vit $\mathrm{C}$ at a constant time interval. The absorbance ratio values obtained at two wavelengths $\left(A_{650} / A_{530}\right)$ was used to quantify the degree of aggregation, $\psi$. The relative extent of the aggregation was defined as $\varepsilon=\psi\left(\mathrm{PFSox}^{-}\right.$@AuNPs $\left.+\mathrm{Vc}\right) /$ $\psi\left(\right.$ PFSox $^{-}$@AuNPs). Fig. 4c shows the values of $\varepsilon$ as a function of varying concentrations of Vit $\mathrm{C}$ used. As is shown, the relative extent of aggregation is a linear function of Vit $\mathrm{C}$ concentration, and provides calibration for the sensing assay. The detection limit (DOL) for Vit $\mathrm{C}$ was found to be $0.01 \mathrm{mM}$. Interestingly, the effect of redox reaction-induced aggregation of $\mathrm{PFS}^{-}$@AuNPs is reversible, which can enable the repeatable use of this colorimetric probe for detecting the redox reaction in a one pot solution. Fig. $4 d$ shows the degree of aggregation of the nanoparticle versus the number of cycles. As can be seen, the sensor remains useable for 13 repeated sensing events. The reason for decreasing the difference as observed with the increasing number of sensing cycles is related to the overall growth of the concentration of the oxidizing and reducing agents in the assay.

Meanwhile, interference factors such as temperature, $\mathrm{pH}$ and the effects of introducing other substances have been studied (ESI, Fig. S3-S5 ${ }^{\dagger}$ ). $\mathrm{PFS}^{-}$is not a $\mathrm{pH}$ sensitive and temperature sensitive polyelectrolyte. The synthesized $\mathrm{PFS}^{-} @ A u N P s$ are stable in $60{ }^{\circ} \mathrm{C}$ aqueous solution or in the solutions with a $\mathrm{pH}$ range from 2 to 10 . In some "inert" bio-substances, such as L-lysine, galactose, and horseradish peroxidase, the particles maintain their redox responsiveness for Vit $\mathrm{C}$ detection.

Other kinds of redox reagents fulfill the following requirements: (a) the oxidant of the ferrocene unit or the reducing agent of the ferrocenium unit; (b) no additional linkage formation between the particles and redox reagents can be detected by this colorimetric method (ESI Fig. S6†). To further verify the usefulness of this sensing system, other experimental studies that focus on enhancing the detection sensitivity and selectivity, and the detection of Vit $\mathrm{C}$ serum are currently underway.

\section{Conclusions}

In summary, we presented an easy and effective one-step process for the preparation of redox-responsive AuNPs capped with stabilizing PFS polyelectrolytes. The PFS polyions featured either positively or negatively charged side-groups. PFS was employed as a reducing agent and as a stabilizer. By judicious design and control of experimental parameters such as the concentration ratio of $\mathrm{Fc}$ and $\mathrm{AuCl}_{4}{ }^{-}$, ionic strength of $\mathrm{NaCl}$, and the type of charges of PFS, PFS@AuNPs were synthesized with a controlled size and stability. In addition, redox-responsive PFS $^{-}$@AuNPs with localized SPR nanostructures provided a unique tunable color and UV-Vis spectral parameters, as well as reversible redox kinetics. We indicate possible applications for the colorimetric detection of other oxidizing and reducing agents in a one-pot solution using Vit $\mathrm{C}$ as a model substance.

\section{Conflicts of interest}

There are no conflicts to declare.

\section{Acknowledgements}

We are grateful to the A*STAR (Agency for Science, Technology and Research), Singapore for providing financial support.

\section{Notes and references}

1 H. Bönnemann and R. M. Richards, Eur. J. Inorg. Chem., 2001, 2455-2480.

2 M. De, P. S. Ghosh and V. M. Rotello, Adv. Mater., 2008, 20, 4225-4241.

3 M. Grzelczak, J. Vermant, E. M. Furst and L. M. LizMarzán, ACS Nano, 2010, 4, 3591-3605.

4 J. M. Pingarrón, P. Yáñez-Sedeño and A. González-Cortés, Electrochim. Acta, 2008, 53, 5848-5866.

5 M. Pumera, S. Sánchez, I. Ichinose and J. Tang, Sens. Actuators, B, 2007, 123, 1195-1205.

6 R. Sardar, A. M. Funston, P. Mulvaney and R. W. Murray, Langmuir, 2009, 25, 13840-13851.

7 Y. Tang and W. Cheng, Sci. Adv. Mater., 2012, 4, 784-797.

8 J. L. West and N. J. Halas, Annu. Rev. Biomed. Eng., 2003, 5, 285-292.

9 M. Haruta and M. Daté, Appl. Catal., A, 2001, 222, 427437.

10 S. Guo and E. Wang, Anal. Chim. Acta, 2007, 598, 181192.

11 P. Zhao, N. Li and D. Astruc, Coord. Chem. Rev., 2013, 257, 638-665.

12 P. Alexandridis, Chem. Eng. Technol., 2011, 34, 15-28.

13 G. Palui, S. Ray and A. Banerjee, J. Mater. Chem., 2009, 19, 3457-3468.

14 J. K. Stolarczyk, A. Deak and D. F. Brougham, Adv. Mater., 2016, 28, 5400-5424.

15 T. Gillich, C. Acikgöz, L. Isa, A. D. Schlüter, N. D. Spencer and M. Textor, ACS Nano, 2013, 7, 316-329.

16 M. S. Yavuz, W. Li and Y. Xia, Chem. - Eur. J., 2009, 15, 13181-13187.

17 W. Schärtl, Nanoscale, 2010, 2, 829-843. 
18 M. Q. Zhu, L. Q. Wang, G. J. Exarhos and A. D. Q. Li, J. Am. Chem. Soc., 2004, 126, 2656-2657.

19 J. M. Tam, J. O. Tam, A. Murthy, D. R. Ingram, L. L. Ma, K. Travis, K. P. Johnston and K. V. Sokolov, ACS Nano, 2010, 4, 2178-2184.

20 Y. Yan, J. I. L. Chen and D. S. Ginger, Nano Lett., 2012, 12, 2530-2536.

21 X. Xie, W. Xu and X. G. Liu, Acc. Chem. Res., 2012, 45, 15111520.

22 E. Tan, J. Wong, D. Nguyen, Y. Zhang, B. Erwin, L. K. Van Ness, S. M. Baker, D. J. Galas and A. Niemz, Anal. Chem., 2005, 77, 7984-7992.

23 G. Song, C. Chen, J. S. Ren and X. G. Qu, ACS Nano, 2009, 3, 1183-1189.

24 X. Liu, Y. Yang and M. W. Urban, Macromol. Rapid Commun., 2017, 38, 1700030.

25 F. Li, J. Lu, X. Kong, T. Hyeon and D. Ling, Adv. Mater., 2017, 29, 1605897.

26 S. T. Camli, F. Buyukserin, C. T. Yavuz and M. S. Yavuz, Mater. Chem. Phys., 2012, 134, 1153-1159.

27 L. Sun, D. Zhao, M. Ding, H. Zhao, Z. Zhang, B. Li and D. Shen, J. Mater. Sci. Technol., 2013, 29, 613-618.

28 P. Alexandridis and M. Tsianou, Eur. Polym. J., 2011, 47, 569-583.

29 K. Kulbaba and I. Manners, Macromol. Rapid Commun., 2001, 22, 711-724.

30 R. L. N. Hailes, A. M. Oliver, J. Gwyther, G. R. Whittell and I. Manners, Chem. Soc. Rev., 2016, 45, 5358-5407.

31 N. G. Connelly and W. E. Geiger, Chem. Rev., 1996, 96, 877-910.

32 D. Jańczewski, J. Song and G. J. Vancso, Eur. Polym. J., 2014, 54, 87-94.

33 D. Fichou, G. Horowitz and F. Garnier, Synth. Met., 1990, 39, 125-131.

34 A. C. Arsenault, H. Míguez, V. Kitaev, G. A. Ozin and I. Manners, Adv. Mater., 2003, 15, 503-507.

35 M. I. Giannotti, H. Lv, Y. Ma, M. P. Steenvoorden, A. R. Overweg, M. Roerdink, M. A. Hempenius and G. J. Vancso, J. Inorg. Organomet. Polym., 2005, 15, 527-540.

36 L.-z. Zhu, W.-b. Zhou and J. Ji, J. Nanopart. Res., 2010, 12, 2179-2187.
37 X. L. Feng, H. R. Wu, X. F. Sui, M. A. Hempenius and G. J. Vancso, Eur. Polym. J., 2015, 72, 535-542.

38 B. Zoetebier, M. A. Hempenius and G. J. Vancso, Chem. Commun., 2015, 51, 636-639.

39 X. Sui, X. Feng, A. Di Luca, C. A. van Blitterswijk, L. Moroni, M. A. Hempenius and G. J. Vancso, Polym. Chem., 2013, 4, 337-342.

40 A. Rapakousiou, C. Deraedt, H. Gu, L. Salmon, C. Belin, J. Ruiz and D. Astruc, J. Am. Chem. Soc., 2014, 136, 13995139958.

41 M. A. Hempenius, F. F. Brito and G. J. Vancso, Macromolecules, 2003, 36, 6683-6688.

42 I. Manners, Adv. Organomet. Chem., 1995, 37, 131168.

43 Q. Yang, L. Wang, W. D. Xiang, C. L. Wang and J. F. Zhou, Prog. Chem., 2006, 18, 290-297.

44 Y. Zhang, Y. Shen, X. Yang, S. Sheng, T. Wang, M. F. Adebajo and H. Zhu, J. Mol. Catal. A: Chem., 2010, 316, 100-105.

45 T. Kim, C.-H. Lee, S.-W. Joo and K. Lee, J. Colloid Interface Sci., 2008, 318, 238-243.

46 M. A. Hempenius, C. Cirmi, J. Song and G. J. Vancso, Macromolecules, 2009, 42, 2324-2326.

47 C. Engelbrekt, P. S. Jensen, K. H. Sorensen, J. Ulstrup and J. Zhang, J. Phys. Chem. C, 2013, 117, 11818-11828.

48 Y. N. Tan, J. Y. Lee and D. I. C. Wang, J. Am. Chem. Soc., 2010, 132, 5677-5686.

49 Y. N. Tan, J. Y. Lee and D. I. C. Wang, J. Phys. Chem. C, 2008, 112, 5463-5470.

50 M. Y. Lin, H. M. Lindsay, D. A. Weitz, R. C. Ball, R. Klein and P. Meakin, Nature, 1989, 339, 360-362.

51 Z. Y. Zhang, S. Maji, A. B. D. Antunes, R. De Rycke, Q. L. Zhang, R. Hoogenboom and B. G. De Geest, Chem. Mater., 2013, 25, 4297-4303.

52 Y. Wang, P. Zhang, X. Mao, W. Fu and C. Liu, Sens. Actuators, B, 2016, 231, 95-101.

53 A. Hayat, B. Gonca and S. Andreescu, Biosens. Bioelectron., 2014, 56, 334-339.

54 G. Wang, Z. Chen and L. Chen, Nanoscale, 2011, 3, 1756-1759. 